\title{
Robot-assisted Extracranial Stereotactic Radiotherapy of Adrenal Metastases in Oligometastatic Non-small Cell Lung Cancer
}

\author{
EREN CELIK, ROBERT SEMRAU, CHRISTIAN BAUES, MAIKE TROMMER-NESTLER, \\ WOLFGANG BAUS and SIMONE MARNITZ \\ University Hospital of Cologne, Department of Radiation-Oncology, Cologne, Germany
}

\begin{abstract}
Aim: The aim of this study was to evaluate the efficacy and toxicity of stereotactic body radiation therapy (SBRT) in the treatment of patients with adrenal metastases in oligometastatic non-small-cell lung cancer (NSCLC). Patients and Methods: Between November 2012 and May 2015, fifteen patients with oligometastatic non-small cell lung cancer and adrenal metastases were treated with the Cyberknife ${ }^{\circledR}$ system. The primary endpoint was local control. Results: The 1-year and 2-year local control rates were 60\% and $46.6 \%$, respectively. The differences in local control for patients with metachronous and synchronous metastases reached statistical significance $(p=0.00028)$. Two-year overall survival of $91.2 \%$ for patients with metachronous metastases was also more favourable compared to patients with synchronous adrenal metastases with $42.8 \%$. Conclusion: Extracranial stereotactic radiotherapy with the Cyberknife $^{\circledR}$ is a safe and non-invasive technique that extends the therapeutic spectrum in the treatment of patients with adrenal metastases in oligometastatic NSCLC.
\end{abstract}

The adrenal gland is a frequent localization of metastases from various cancers. The incidence of these metastases from autopsies ranges between $13-35 \%$ (1). Lung cancer is the second most common site of origin (2). Oncological management contains a diversity of treatment options such as, surgical resection, local ablative treatments (3-5), chemoembolization or palliative chemotherapy, which remains standard for most patients with metastatic disease (6).

Correspondence to: Eren Celik, Department of Radiation-Oncology, University Hospital of Cologne, Kerpener Straße 62, 50937 Cologne, Germany. Tel: +49 2214785495, Fax: +49 2214785097, email: eren.celik@uk-koeln.de

Key Words: Adrenal metastases, non-small cell lung cancer, oligometastases, hypofractionated stereotactic radiotherapy, metastasis, Cyberknife ${ }^{\circledR}$.
Several studies have demonstrated prolonged survival after an adrenal metastasectomy in selected patients with isolated adrenal metastases. A pooled analysis of more than 600 patients showed a 2-year overall survival (OS) for patients undergoing adrenalectomy for adrenal gland metastases of $46 \%$ (7). In the past, fractionated radiation therapy has been widely used with and was mainly considered as a symptomatic palliative treatment option (8, 9). The development of stereotactic body radiotherapy (SBRT) and the improved understanding of oligometastatic disease, as a biologically distinct entity led to a paradigm shift in the role of radiotherapy for the treatment of patients with one to five distant metastases (10-13). In those patients local therapies are considered as a reasonable option to prolong progression- free survival.

Stereotactic radiosurgery (SBRT) allows for local application of high biologically effective doses (BED) with steep dose gradients towards the surrounding normal tissue using a single or limited number of dose fractions causing low toxicity (14). Usually, SBRT provides much higher BEDs compared to conventional treatments and has been used as a non-invasive alternative to the standard of care in highly selected patients $(15,16)$. Especially patients with oligometastatic disease benefit from local treatment modalities that allow long-term local control (17). There is increasing evidence that lung cancer patients with oligometastases benefit from aggressive local therapies, in the form of SBRT or surgery, with better outcomes and prolonged survival (18). Compared to surgery, a matched pair analysis including 62 patients has shown that there was no significant difference of outcome and survival after SBRT and laparoscopic adrenalectomy in patients with isolated adrenal metastases (19).

For SBRT in patients with adrenal metastases, 1- and 2year local control (LC) rates range from $44-100 \%$ and from $27-100 \%$, respectively. In most of the studies a 1- and 2-year LC of $>70 \%$ could be achieved (20-22). As for primary NSCL, there is a dose- response relationship. Publications who reported on BEDs $>85$ Gy showed an even better 2-year 
local control of at least $90 \%(23,24)$. Patients with oligometastatic metachronous disease with a progression free interval of at least six months showed better outcomes with a mean overall survival of 44 months (25) and one year OS of $>90 \%$, respectively (26).

Generally, SBRT of adrenal metastases is well tolerated. Mostly, grade 1-2 gastrointestinal toxicity is reported. Duodenal ulcers were reported after BED to the tumor of 60 and $72 \mathrm{~Gy}$, respectively, indicating the questionable validity of dose-volume-parameters $(25,26)$. The incidence of symptomatic adrenal insufficiency seems to be low (27). One clinical case of adrenal insufficiency after SBRT for bilateral adrenal metastases has been reported (28).

Normal tissue tolerance and precise, continuously tumor motion tracking remain substantial aspects when delivering high ablative doses of radiation. Katoh et al. demonstrated that adrenal glands can move to all directions (29). Therefore, protecting critical organs surrounding the adrenal gland requires an effective way of tumor tracking. We used an image-guided robotic radiotherapy system, which is capable of analysing and adjusting tumor motion as well as breathing patterns of the treated patients.

This study reports on our single-institution outcome after robot-based SBRT of adrenal metastases from oligometastatic non-small cell lung cancer with one of the largest homogenous collective of patients in terms of fractionation schedule and clinical stage at time of treatment.

\section{Patients and Methods}

Patients characteristics. Consecutive eligible patients with adrenal metastases were selected to be included in this study under following inclusion criteria: a) patients of any age b) the presence of oligometastases ( 1 to 5 lesions) from histologically-proven nonsmall cell lung cancer c) unilateral adrenal metastasis, which was confirmed after biopsy of an interventional radiologist or by clinical diagnosis d) contraindication to surgical treatment due to poor general condition or restricted heart and lung function e) a Performance Status $>60 \%$ (Karnofsky score) f) stable disease concerning the primary g) no previous in-field radiation. Treatment response was assessed using the Response Evaluation and Criteria in Solid tumors 1.1 (30), while toxicity was evaluated with the Common Terminology Criteria for Adverse Events v4.0 criteria. Based on prior surgical and radiooncological studies that showed improved treatment response rates dependent on disease-free interval, defined as the time from diagnosis of non-small cell lung cancer to diagnosis of adrenal metastasis, we determined adrenal lesions as either synchronous or metachronous. Synchronous metastases were defined as such, if they were diagnosed $\leq 6$ months from time of original diagnosis and metachronous after six months from original diagnosis. Seven patients had synchronous metastases and eight patients had metachronous adrenal metastases. Patient characteristics are summarized in Table I. Patients treated between November 2012 and May 2015 were included in the analysis, and medical records of patients with adrenal metastases from oligometastatic non-small cell lung cancer were retrospectively reviewed. Fifteen patients with metastatic adrenal lesions were treated in six fractions up to a total dose of 42 Gy with the SBRT CyberKnife ${ }^{\circledR}$ system.

Treatment. Radiotherapy was delivered by using the CyberKnife ${ }^{\circledR}$, a stereotactic radiation therapy system consisting of a 6-MV photon medical accelerator mounted on six degrees of freedom robotic arm with integrated real time image-guidance. The beams are formed either by fixed collimators or a motor-driven device, the opening ranging from five up to $60 \mathrm{~mm}$. Two X-ray imaging cameras are mounted with their beams at a 90-degree angle around the patient and allow continuous execution of $\mathrm{x}$-ray images and registration of these to digitally reconstructed radiographs provided by the treatment planning system. This registration uses either bony structures $(6 \mathrm{D}$ Skull, Xsight ${ }^{\circledR}$ Spine) or fiducial markers (Fiducial) or, in the case of lung, the tumor itself (Xsight ${ }^{\circledR} \mathrm{Lung}$ ). In a first step, this information is used to position the patient via couch movement. During treatment, this target locating system facilitates the repositioning of the robotic arm to compensate for displacements up to $10 \mathrm{~mm}$ with a resolution of $0.1 \mathrm{~mm}$. To manage respiratory motion, the $\mathrm{X}$-ray images are combined simultaneously with the breathing pattern of the patient which is derived from the movement of LEDs positioned on the patient's chest (Synchrony ${ }^{\circledR}$ ). Radiation treatment was planned using CT-based 3-D planning software (Accuray Multiplan 4.5 and 4.6) after prior performance of a computer tomography with $1 \mathrm{~mm}$ slice thickness and positioning of the patients in a vacuum mattress. The adrenal lesions visible on planning CT were taken as the gross tumor volume (GTV). GTV was expanded by $4 \mathrm{~mm}$ to create the planning target volume (PTV). The dose of 7 Gy per fraction was prescribed to an isodose line of $65 \%$. The consistent fractionation was six fractions of $7 \mathrm{~Gy}$ for all fifteen patients, who were treated in this series. Treatment was delivered in a median treatment time of 14 days (range $=11-18$ days), with a treatment session on each other working day.

Follow-up. Follow-up included physical examination and treatmentrelated anamnesis and was performed first at 6 weeks after completion of treatment and subsequently every 3 months for 2 years. Abdominal CT with i.v. contrast medium was performed with each 3-month visit. Treatment response was evaluated using the RECIST 1.1 criteria by the attending radiation oncologist after review by at least one radiologist. In accordance to these criteria, complete response (CR) was defined as complete disappearance of tumor volume or only remaining fibrosis, partial response (PR) determined as tumor shrinkage of at least $30 \%$, stable disease (SD) as no quantifiable difference and progressive disease (PD) as a tumor increase of at least $20 \%$. Local control was defined as CR, PR or SD during follow-up imaging. Acute toxicity was evaluated with the CTCAE v4.0. Pain relief and need for analgesics were documented.

Statistics. Survival and local control rates were calculated from the time of initiation of SBRT using the Kaplan-Meier method. Differences in overall survival, local control and disease-free survival were evaluated using a log-rank test. $p$-Values $\leq 0.005$ were considered statistically significant. The primary endpoint was local control, defined as no growth of the treated lesion. Distant failure was defined as failure outside of the treated adrenal gland. Furthermore, disease free survival was determined as progression of an existing target lesion, appearance of new lesions within the 
Table I. Patients characteristics.

\begin{tabular}{lc}
\hline Variable & $\mathrm{N}(\%)$ \\
\hline Median Age, years (range) & $70(54-84)$ \\
Gender & $8(53)$ \\
$\quad$ Male & $7(47)$ \\
Female & $80(60-100)$ \\
Median Karnofsky Performance Score (range) & \\
Histology N (\%) & $10(67)$ \\
Adenocarcinoma & $3(20)$ \\
Squamous cell carcinoma & $2(13)$ \\
Giant cell carcinoma & \\
Giant cell carcinoma & \\
Time (months) from diagnosis to SBRT & 2.3 \\
Median & $1.2-4.5$ \\
Range & $5(33 \%)$ \\
Prior chemotherapy within 6 months & $6(40 \%)$ \\
More than 3 cycles chemotherapy prior to SBRT & \\
Status of oligometastatic disease & $9(60 \%)$ \\
Controlled primary, adrenal metastasis only & $6(40 \%)$ \\
Controlled primary, adrenal + others* & \\
\hline
\end{tabular}

*Other metastases ( $\leq 5$ in total).

same organ, distant relapse or death due to cancer. All statistical analyses were performed using IBM SPSS version 21 .

\section{Results}

Patients characteristics. Fifteen patients (8 males, 7 females) were treated between November 2012 and May 2015 at the University Hospital of Cologne. The median age at time of treatment was 70 years (range $=54-84$ years). Histological classification of treated patients was adenocarcinoma in ten patients, squamous cell carcinoma in three patients and giant cell carcinoma in two cases. The median time from adrenal metastases diagnosis to CyberKnife ${ }^{\circledR}$ treatment was 2.36 months (range=1.25-4.5 months). Thirty-three percent (5 patients) received prior chemotherapy within the last six months, whereas six patients $(40 \%)$ received more than three cycles of chemotherapy regimen prior to SBRT. Nine patients $(60 \%)$ of the treated oligometastatic patients had one up to three metastatic lesions, whereas six patients (40\%) presented with four up to five metastatic lesions at the start of SBRT. None of the treated patients had received prior radiotherapy. Treatment characteristics are shown in Table II.

Treatment characteristics. All treated patients received a total nominal dose of 42 Gy in six fractions using the CyberKnife ${ }^{\circledR}$ treatment system. Median overall treatment time was 14 days (range $=11-18$ days). Consequently, the biologically equivalent dose (BED) was consistently $71.4 \mathrm{~Gy}$ with an alpha/beta ratio of $10 \mathrm{~Gy}$ for the tumor used to calculate it. Treatment dose of 7 Gy was administered to a median prescription isodose line
Table II. Treatment characteristics and dose constraints.

\begin{tabular}{lc}
\hline Feature & Value \\
\hline Dose (Gy) & 42 \\
Fractions (n) & 6 \\
Fraction size (Gy) & 7 \\
BED10 & 71.4 \\
GTV $\left(\mathrm{cm}^{3}\right)$ & \\
$\quad$ Median & 36.1 \\
$\quad$ Range & $11.1-88.4$ \\
PTV (cm $\left.{ }^{3}\right)$ & 74.4 \\
$\quad$ Median & $37.4-137.2$ \\
$\quad$ Range & \\
Used Monitor Units & 49983 \\
$\quad$ Median & $42,371-71,148$ \\
$\quad$ Range & \\
Beams & 402.5 \\
$\quad$ Median & $397-406$ \\
Range & \\
Isodose line (\%) & 68.7 \\
Median & $65-73$ \\
Range & \\
\hline
\end{tabular}

GTV, Gross tumor volume; PTV, planning target volume; Gy, gray. BED10, biological equivalent dose for an alpha/beta ratio of 10 .

Table III. Critical organ constraints.

\begin{tabular}{ll}
\hline Organ & Constraints \\
\hline Ipsilateral kidney & V20 Gy $\leq 40 \%$ \\
Bilateral kidney & V15 Gy $<33 \%$ \\
Spinal cord & $\mathrm{D}_{\max }<27 \mathrm{~Gy}$ \\
Stomach/duodenum & $0.5 \mathrm{cc}<30 \mathrm{~Gy}$ \\
\hline
\end{tabular}

that covered $68.7 \%$ of the PTV with median GTV coverage of $98.5 \%$. Median gross tumor volume treated was $36.14 \mathrm{~cm}^{3}$ (range $=11.17-88.43 \mathrm{~cm}^{3}$ ) and median planning tumor volume treated was $74.45 \mathrm{~cm}^{3}$ (range $=37.4-137.29 \mathrm{~cm}^{3}$ ). Critical organ dose constraints are detailed in Table III.

Treatment response. Median follow-up was 24 months (range=8-48 months) and evaluation of RECIST-based twodimensional response was carried out based on an abdominal $\mathrm{CT}$ that was performed after a minimum period of 3 months after completion of CyberKnife ${ }^{\circledR}$ SBRT. Complete response was observed in no patient, partial response in eight patients and stable disease in one patient. Six patients showed progressive disease after RECIST-based assessment, resulting in a response rate $(\mathrm{CR}+\mathrm{PR})$ of $53.3 \%$. All patients 
with progressive disease had synchronous adrenal metastases within $\leq 6$ months from time of first diagnosis.

Palliative response. Three out of fifteen patients had symptomatic adrenal metastases prior to SBRT, which were rated as moderate on the visual scale $(\leq 5)$. Cyberknife ${ }^{\circledR}$ treatment was applied for the remaining twelve asymptomatic patients within the scope of radiooncological management of oligometastatic disease with solitary progression of adrenal metastases. Two of the symptomatic patients experienced significant pain relief, while one patient achieved moderate symptomatic palliation. Response was associated with decreased usage of analgesics.

Local control and patient survival. The 1-year and 2-year Kaplan-Meier local control at a median follow-up of 24 months was $60 \%$ and $46.6 \%$, respectively. The median local control was 11 months. The median post-therapy overall survival rates at one and two years were $93.3 \%$ and $66.6 \%$, respectively with a median overall survival of 17.3 months. Disease-free survival 1-year and 2-years after completion of SBRT was $60 \%$ and $46.6 \%$, respectively. The median disease-free survival was 10.5 months. All the deceased patients $(n=5)$ died of progressive distant metastatic disease.

Patients with metachronous adrenal metastases (i.e., diagnosed after six months from original diagnosis) had statistically significant better outcomes regarding local control, overall survival and disease-free survival compared with the whole cohort including patients with synchronous and metachronous adrenal metastases (Figure 1). The 1-year and 2-year local control was constantly $87.5 \%$ compared to $14.2 \%$ and $0 \%$ for those with synchronous metastases $(p=0.00028)$. Median disease-free survival for patients with metachronous metastases was 17.2 months in comparison with 3.0 months for those with synchronous metastases $(p=0.0003)$. There was also a statistically significant better outcome for the metachronous group regarding Kaplan-Meier overall survival at 1-year and 2-years with $100 \%$ and $91.2 \%$, respectively by contrast with $85.7 \%$ and $42.8 \%$ for the synchronous group $(p=0.05)$. A median overall survival of 20.3 months was observed for patients with synchronous metastases, whereas 13.5 months were analysed for patients with adrenal metastases diagnosed $\leq 6$ months from time of original diagnosis.

In summary, eight patients presented local failure of the treated adrenal metastases in the further course of follow-up. At the end of follow-up analysis, five of all treated patients died. No death was related to complications with adrenal metastases. Progression of the primary disease, non-small cell lung cancer, resulted into three cancer related deaths. Two patients died of cerebral complications due to brain metastasis.
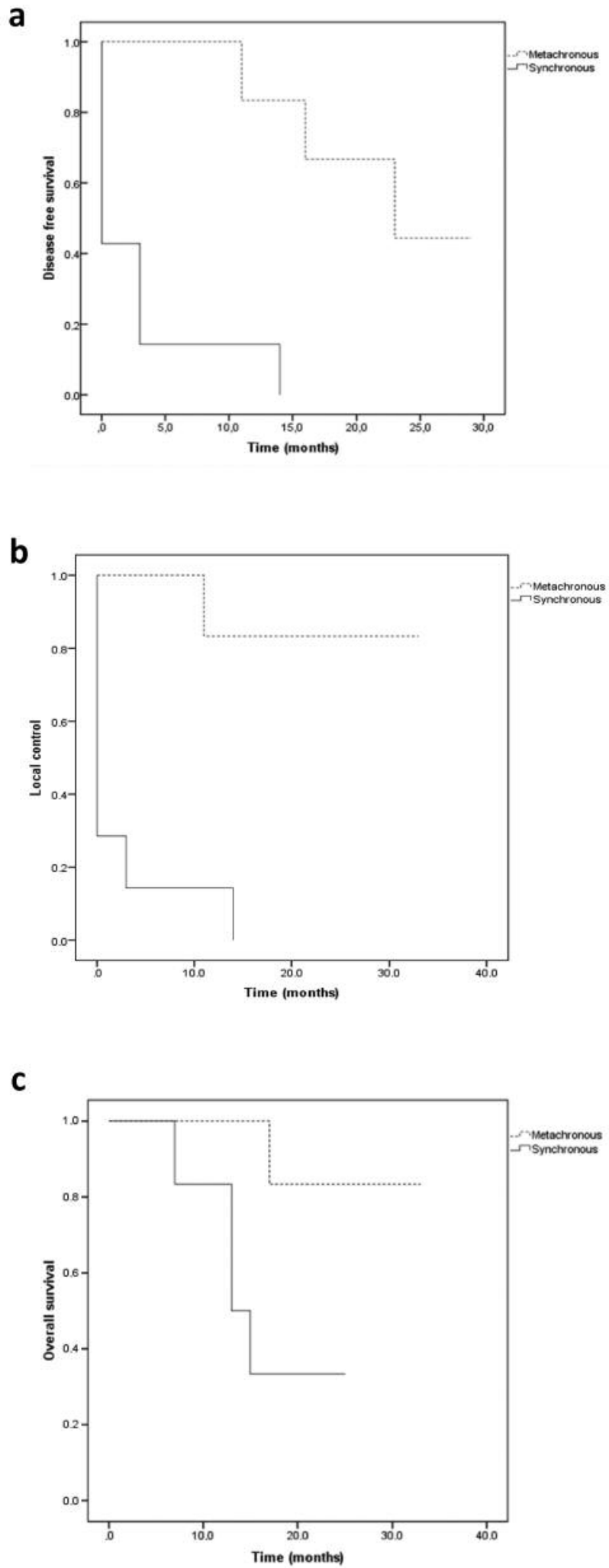

Figure 1. Survival curves of metachronous and synchronous adrenal metastases. a: Disease-free survival, $b$ : local control, $c$ : overall survival. 
Toxicity. No grade $\geq 3$ early or late side-effects according to CTCAE v4.0 were observed during SBRT or at the end of follow up. Reported acute adverse effects included nausea and loss of appetite in three patients that were treated adequately with oral anti-emetics. Within a median follow up of 24 months after completion of SBRT, no grade 3 or higher late toxicities were observed.

\section{Discussion}

The aim of this retrospective study was to evaluate the efficacy of SBRT using the Cyberknife ${ }^{\circledR}$ system in the treatment of oligometastatic NSCLC with adrenal metastases. Our results demonstrated that SBRT using the Cyberknife ${ }^{\circledR}$ can achieve local control without severe toxicity for these patients and confirm findings of previous studies about the beneficent role of SBRT for adrenal metastases (20-26). Nevertheless, current studies existing on SBRT of adrenal metastases are very heterogeneous in terms of patient collectives and dose fractionation schedules, which hampered efforts to define a uniform treatment regimen. We therefore present our single-institution series with, as to our knowledge, one of the largest homogenous series in terms of patient selection (primary tumor, disease extension, clinical stage) and treatment characteristics (dose and fractionation schedule) after robotic SBRT of adrenal metastases from oligometastatic non-small cell lung cancer.

A recently published review from Ippolito et al. showed that local control rates of adrenal metastases using SBRT are promising, with rates ranging from $44 \%$ to $100 \%$ at 1 year and $27 \%$ to $100 \%$ at 2 years (31). Our study coincides with the published SBRT data demonstrating that this treatment modality achieves good local control for all our patients. We had 1-year and 2-year local control rates of $60 \%$ and $46.6 \%$, respectively. However, when considering our subgroups of metachronous and synchronous adrenal metastases, we achieved considerably higher LC rates for those patients with metachronous metastases with $87.5 \%$ at 1 and 2 years. Given the limited number of published studies about SBRT of adrenal metastases, no significant effect between LC and manifestation of metastases was described yet. Although the patients in our study were far more homogenous in terms of histology, primary cancer and clinical stage at the time of SBRT, these results are consistent with previous studies showing that general outcome of patients with adrenal metastases, receiving SBRT or in case of surgical studies adrenalectomy, may depend on metastatic spread in terms of appearance from time of original diagnosis $(25,26,32)$. Further studies with larger and more homogenous patient collectives are needed to elucidate a possible correlation between local control and a higher proportion of synchronous manifestation of metastases.
In addition, BED and, therefore, dose and fractionation schedules seem to be important prognostic factors in terms of local control (33). Previous studies about SBRT of adrenal metastases showed a wide heterogeneity as for chosen dose and fractionation schedules. Most studies used hypofractionated SBRT with a nominal total dose range of 25-48 Gy administered in fractions of 3 up to 18 $(\mathrm{BED}=22.4-132, \alpha / \beta=10)$, with a total number of 5 and 3 fractions delivered most commonly (in both cases 5/12, $41.6 \%$ ). All our patients received a total nominal dose of 42 Gy in six fractions using the CyberKnife ${ }^{\circledR}$ treatment system. Consequently, our BED was consistently $71.4 \mathrm{~Gy}(\alpha / \beta=10)$ and thus fits in well with dose and fractionation schedules used in previous studies about SBRT of adrenal metastases. Of note, CyberKnife ${ }^{\circledR}$ SBRT was applied in only two of the considered studies, with a dose range of 13-36 Gy $(B E D=29.9-79.2, \alpha / \beta=10)$. Three fractions were most frequently administered (10/23 treated lesions, 43\%) (22, $33,34)$.

As for primary lung cancer, there is a proven correlation between local control and the prescribed $\operatorname{BED}(35,36)$. A clear dose-response relationship has been observed also in several studies about SBRT for adrenal metastases. Studies that showed 1- year local control rates lower than $70 \%$ used BEDs $<60$ Gy $(21,22,37)$. In contrast, publications who reported on BEDs $>85$ Gy showed considerably better 2-year local control rates of at least $90 \%$ (23-25). In this context, our results on local control are not consistent with observed patterns of previous reports. Even though we applied a BED of 71.4, our results on local control are inferior to hitherto published studies about SBRT of adrenal metastases with 1year and 2-year local control rates of $60 \%$ and $46.6 \%$, respectively. Differences could be explained by our bigger subgroup of patients with synchronous metastases (46.6\%). Local control rates of $87.5 \%$ at 1-year in our subgroup with metachronous metastases, on the other hand, were excellent. Guiou et al., for example, treated 10 adrenal metastases with a portion of $50 \%$ of synchronous metastases. Local control rates were $44 \%$ and $14 \%$ at 1- and 2-year, respectively (37). Whereas in the study by Holy et al. patients with metachronous, isolated metastases $(72.2 \%)$ had a local control of $77 \%$ after a median follow-up of 21 months (38). Proper patients' selection influences oncologic results.

The median overall survival after SBRT of adrenal metastases ranged from 7-23 months, with 1- and 2-year overall survival rates ranging from $39.7-90 \%$ and $13-53 \%$, respectively. Despite the high ratio of generally poor prognostic features in our patients, such as advanced clinical stage and high proportion of synchronous metastases, the median overall survival of our patient collective with adrenal metastases receiving CyberKnife ${ }^{\circledR}$ SBRT treatment was 17.3 months with median post-therapy overall survival rates at one and two years of $93.3 \%$ and $66.6 \%$. These 
results are comparable with the rates previously reported for patients with adrenal metastases from various primary sites who received SBRT. Furthermore, our results agree with those from the study conducted by Oshiro et al., who reported of 19 patients with adrenal metastases from NSCLC from which 11 were treated with SBRT. Improved survival was found in patients with metachronous metastases with 1- and 2-year overall survival rates of $83.3 \%$ and $55.6 \%$, as opposed to patients with synchronous metastases of $35 \%$ and $0 \%$ (25). The awareness for existing subgroups of patients with a disparate prognosis is of crucial importance in terms of patient selection and subsequently further treatment decisions, especially for patients with oligometastatic disease (40).

There was no grade $\geq 3$ or higher late toxicities in our study. The most common acute toxicities were nausea and loss of appetite during the treatment period. Oshiro et al. reported one patient with grade 2 duodenal ulcers, who was treated with a histamine-2 receptor antagonist (25). Holy et $a l$, as well had one patient with duodenal and one with gastric ulcers (38). There were no SBRT-related grade 4 events in previous studies.

Due the fact that all current studies on SBRT of adrenal metastases are retrospective observational studies, patients' selection, therapeutic concepts and oncologic outcome differ considerable. Since it has been unlikely to implement a prospective study, retrospective data underlie the importance of patients' selection. Patients with prescribed BED>70 Gy and metachronous disease may have a larger benefit from CyberKnife ${ }^{\circledR}$ SBRT. Treatment response appears to be satisfactory with SBRT and even excellent in our subgroup of patients with metachronous adrenal metastases. We used one fractionation scheme for all our patients. Our further studies will investigate the effects of a higher BED (>85 Gy) on local control in a homogenous patient collective.

\section{Conclusion}

Overall, robotic SBRT is an effective treatment modality with excellent local control rates and low toxicity for adrenal metastases in patients with oligometastatic NSCLC. While optimal dose is under discussion, additional studies and shared data bases of different user with more distinct characteristics in terms of patient selection, dose and fractionation schedules should help to make summarizing appraisals about the best use of SBRT for adrenal metastases. Better outcomes of particular subgroups should also be considered to define those patient collectives which benefit most likely from SBRT.

\section{Conflicts of Interest}

The Authors state that there are no conflicts of interest.

\section{References}

1 Abrams HL, Spiro R and Goldstein N: Metastases in carcinoma; analysis of 1000 autopsied cases. Cancer 3: 74-85, 1950.

2 Lam KY and Lo CY: Metastatic tumours of the adrenal glands: a 30-year experience in a teaching hospital. Clin Endocrinol 56: 95-101, 2002.

3 Strong VE, D’Angelica M, Tang L, Prete F, Gönen M, Coit D, Touijer KA, Fong $\mathrm{Y}$ and Brennan MF: Laparoscopic adrenalectomy for isolated adrenal metastasis. Ann Surg Oncol 14: 3392-3400, 2007.

4 Karagkiouzis G, Spartalis E, Moris D, Patsouras D, Athanasiou A, Karathanasis I, Verveniotis A, Konstantinou F, Kouerinis IA, Potaris K, Dimitroulis D and Tomos P: Surgical Management of Non-Small Cell Lung Cancer with Solitary Hematogenous Metastases. In Vivo 31: 451-454, 2017.

5 Porte H, Siat J, Guibert B, Lepimpec-Barthes F, Jancovici R, Bernard A, Foucart A and Wurt A: Resection of adrenal metastases from non-small cell lung cancer: a multicenter study. Ann Thorac Surg 71: 981-985, 2001.

6 Wood BJ, Abraham J, Hvizda JL, Alexander HR and Fojo T: Radiofrequency ablation of adrenal tumors and adrenocortical carcinoma metastases. Cancer 97: 554-560, 2003.

7 Gunjur A, Duong C, Ball D and Siva S: Surgical and ablative therapies for the management of adrenal 'oligometastases' - A systematic review. Cancer Treat Rev 40: 838-846, 2014.

8 Soffen EM, Solin LJ, Rubenstein JH and Hanks GE: Palliative radiotherapy for symptomatic adrenal metastases. Cancer 65 : 1318-1320, 1990.

9 Short S, Chaturvedi A and Leslie MD: Palliation of symptomatic adrenal gland metastases by radiotherapy. Clinical Oncology 8 : 387-389, 1996.

10 Corbin KS, Hellman S and Weichselbaum RR: Extracranial Oligometastases: A subset of metastases curable with stereotactic radiotherapy. J Clin Oncol 31: 1384-1390, 2013.

11 Weichselbaum RR and Hellman S: Oligometastases revisited. Nature Reviews Clinical Oncology 8: 378-382, 2011.

12 Salama JK, Hasselle MD, Chmura SJ, Malik R, Mehta N, Yenice KM, Villaflor VM, Stadler WM, Hoffman PC, Cohen EEW, Connell PP, Haraf DJ, Vokes EE, Hellmann S and Weichselbaum RR: Stereotactic body radiotherapy for multisite extracranial oligometastases. Final report of a dose escalation trial in patients with 1 to 5 sites of metastatic disease. Cancer 118: 2962-2970, 2012.

13 Hellman S and Weichselbaum RR: Oligometastases. J Clin Oncol 13: 8-10, 1995.

14 Potters L, Kavanagh B, Galvin JM, Hevezi JM, Janjan NA, Larson DA, Mehta MP, Ryu S, Steinberg M, Timmerman R and Welsh JS: American Society for Therapeutic Radiology and Oncology (ASTRO) and American College of Radiology (ACR) practice guideline for the performance of stereotactic body radiation therapy. Int J Radiat Oncol Biol Phys 76: 326-332, 2010.

15 Rusthoven KE, Kavanagh BD, Burri SH, Chen C, Cardenes H, Chidel MA, Pugh TJ, Kane M, Gaspar LE and Schefter TE: Multi-institutional phase I/II trial of stereotactic body radiation therapy for lung metastases. J Clin Oncol 27: 1579-1584, 2009.

16 Janssen S, Kaesmann L, Rudat V and Rades D: Stereotactic body radiotherapy provides excellent long-term local control of very few Lung Metastases. In Vivo 30: 155-157, 2016. 
17 Tree AC, Khoo VS, Eeles RA, Ahmed M, Dearnaley DP, Hawkins MA, Huddart RA, Nutting CM, Ostler PJ and van As NJ: Stereotactic body radiotherapy for oligometastases. Lancet Oncol 31: 28-37, 2013.

18. Patel PR, Yoo, DS, Niibe Y, Urbanic JJ and Salama JK: A call for the aggressive treatment of oligometastatic and oligo-recurrent non-small cell lung cancer. Pulm Med 2012: 480961, 2012.

19. Arnaud A, Caiazzo R, Claude L, Zerrweck C, Carnaille B, Pattou F and Carrie C: Stereotactic Body Radiotherapy vs. Surgery for Treatment of Isolated Adrenal Metastases: A Matched Pair Analysis Including 62 Patients. Int J Radiat Oncol Biol Phys 81: 89, 2011.

20. Torok J, Wegner RE, Burton SA and Heron DE: Stereotactic body radiation therapy for adrenal metastases: a retrospective review of a noninvasive therapeutic strategy. Future Oncol 7: 145-151, 2011.

21 Scorsetti M, Alongi F, Filippi AR, Pentimalli S, Navarria P, Clerici E, Castiglioni S, Tozzi A, Reggiori G, Mancosu P and Ricardi U: Long-term local control achieved after hypofractionated stereotactic body radiotherapy for adrenal gland metastases: a retrospective analysis of 34 patients. Acta Oncol 51: 618-623, 2012.

22 Chawla S, Chen Y, Katz AW, Muhs AG, Philip A, Okunieff P and Milano MT: Stereotactic body radiotherapy for treatment of adrenal metastases. Int J Radiat Oncol Biol Phys 75: 71-75, 2009.

23 Ahmed KA, Barney BM, Macdonald OK, Miller RC, Garces YI, Laack NN, Haddock MG, Foote RL and Olivier KR: Stereotactic body radiotherapy in the treatment of adrenal metastases. Am J Clin Oncol 36: 509-513, 2013.

24 Casamassima F, Livi L, Masciullo S, Menichelli C, Masi L, Meattini I, Bonucci I, Agresti B, Simontacchi G and Doro R: Stereotactic radiotherapy for adrenal gland metastases: university of Florence experience. Int J Radiat Oncol Biol Phys 82: 919923, 2012.

25 Oshiro Y, Takeda Y, Hirano S, Ito H and Aruga T: Role of radiotherapy for local control of asymptomatic adrenal metastasis from lung cancer. Am J Clin Oncol 34: 249-253, 2011.

26 Rudra S, Malik R, Ranck MC, Farrey K, Golden DW, Hasselle MD, Weichselbaum RR and Salama JK: Stereotactic Body Radiation Therapy for Curative Treatment of Adrenal Metastases. Technol Cancer Res Treat 12: 217-224, 2013.

27 Redman BG, Pazdur R, Zingas AP and Loredo R: Prospective Evaluation of Adrenal Insufficiency in Patients with Adrenal Metastasis. Cancer 60: 103-107, 1987.

28 Wardak Z, Meyer J, Ghayee H, Wilfong L and Timmerman R: Adrenal insufficiency after stereotactic body radiation therapy for bilateral adrenal metastases. Pract Radiat Oncol 5: 177-181, 2015.

29 Katoh N, Onimaru R, Sakuhara Y, Abo D, Shimizu S, Taguchi H, Watanabe Y, Shinohara N, Ishikawa M and Shirato H: Realtime tumor-tracking radiotherapy for adrenal tumors. Radiother Oncol 87: 418-424, 2008.
30 Eisenhauer EA, Therasse P, Bogaerts J, Schwartz LH, Sargent D, Ford R, Dancey J, Arbuck S, Gwyther S, Mooney M, Rubinstein L, Shankar L, Dodd L, Kaplan R, Lacombe D and Verweij J: New response evaluation criteria in solid tumours: revised RECIST guideline (version 1.1). Eur J Cancer 45: 228247, 2009.

31 Ippolito E, D'Angelillo RM, Fiore M, Molfese E, Trodella L and Ramella S: SBRT: A viable option for treating adrenal gland metastases. Rep Pract Oncol Radiother 20: 484-490, 2015.

32 Tanvetyanon T, Robinson LA, Schell MJ, Strong VE, Kapoor R, Coit DG and Bepler G: Outcomes of adrenalectomy for isolated synchronous versus metachronous adrenal metastases in nonsmall-cell lung cancer: A systematic review and pooled analysis. J Clin Oncol 26: 1142-1147, 2008.

33 Desai A, Rai H, Haas J, Witten M and Schneider JG: A retrospective review of cyberknife stereotactic body radiotherapy for adrenal tumors (primary and metastatic): Winthrop university hospital experience. Front Oncol 5: 185, 2015.

34 Karagounis IV, Skourti EK, Liousia MV and Koukourakis MI: Assessment of Radiobiological $\alpha / \beta$ Ratio in Lung Cancer and Fibroblast Cell Lines Using Viability Assays. In Vivo 31: 175179, 2017.

35 Onishi H, Araki T, Shirato H, Nagata Y, Hiraoka M, Gomi K, Yamashita T, Niibe Y, Karasawa K, Hayakawa K, Takai Y, Kimura T, Hirokawa Y, Takeda A, Ouchi A, Hareyama A, Kokubu M, Hara R, Itami $\mathrm{J}$ and Yamada K: Stereotactic hypofractionated high-dose irradiation for stage I nonsmall cell lung carcinoma. Cancer 101: 1623-1631, 2004.

36 Filippi AR, Franco P and Ricardi U: Is stereotactic ablative radiotherapy an alternative to surgery in operable stage I nonsmall cell lung cancer? Rep Prac Oncol Radiother 19: 275-279, 2014.

37 Guiou M, Mayr NA, Kim EY, Williams T and Lo SS: Stereotactic body radiotherapy for adrenal metastases from lung cancer. J Radiat Oncol 1: 155-63, 2012.

38 Holy R, Piroth M, Pinkawa M and Eble MJ: Stereotactic body radiation therapy (SBRT) for treatment of adrenal gland metastases from non-small cell lung cancer. Strahlenther Onkol 187: 245-251, 2011.

39 Griffioen GH, Toguri D, Dahele M, Warner A, De Haan PF, Rodrigues GB, Slotman BJ, Yaremko BP, Senan S and Palma DA: Radical treatment of synchronous oligometastatic non-small cell lung carcinoma (NSCLC): patient outcomes and prognostic factors. Lung cancer 82: 95-102, 2013.
Received June 5, 2017

Revised July 5, 2017

Accepted August 5, 2017 